\title{
A Note on Intertemporal Discounting and Nudging
}

\author{
Ahmad S. Taleb ${ }^{1} \&$ Ameen Talib ${ }^{2, *}$ \\ ${ }^{1}$ University of Edinburgh, United Kingdom \\ ${ }^{2}$ School of Business, Singapore University of Social Sciences, Singapore \\ *Correspondence: School of Business, Singapore University of Social Sciences, Singapore. E-mail: \\ ameentalib@suss.edu.sg
}

Received: December 17, 2019

Accepted: December 27, 2019 Online Published: February 17, 2020

doi:10.5430/mos.v6n2p24

URL: https://doi.org/10.5430/mos.v6n2p24

\begin{abstract}
Several challenges and rising costs are facing the healthcare industry today. Some of these health costs are a direct consequence of lifestyle choices such as unhealth diets, obesity and smoking. These challenges can be managed if policy makers have the tools to influence and alter people's behaviour. The conventional tools used for influencing behaviour include legislation, regulation and information provision. Recently, interest has been shown in policies that 'nudge' people in particular directions.
\end{abstract}

Keywords: nudge, intertemporal discounting, health choices, behaviour

\section{A Note on Intertemporal Discounting and Nudging}

The world is ageing and rising health costs are a concern. Some of these health costs are a direct consequence of lifestyle choices such as unhealth diets, obesity and smoking. A number of the challenges we are facing in the healthcare industry is due to current (moral hazards) behaviour. These challenges could be resolved if we were successful in finding a way to change these behaviours. The conventional tools used for influencing behaviour include legislation, regulation and information provision. Recently, interest has been shown in policies that 'nudge' people in particular directions; drawing on major advances in our understanding that behaviour is influenced by the context and situation within which it is placed. Insights from across the behavioural sciences and particularly behavioural economics provide us with a powerful set of new and refined policy tools to use when trying to influence health-related behaviours.

Traditional theories focused on utility and the discounted utility theory. One problem with this is the underlying assumption of rational behaviour. The rational choice theory assumes individuals discount all future utilities equally, however Stotrz (1955) demonstrated that individuals actually follow a hyperbolic function. Lately interventions sim to improve absolute welfare without restricting freedom of choice. This "choice architecture" characterises the notion that by altering the environments where choices are made, behaviour can be modified in predictable ways. This is also called nudging.

This paper attempts to explain the working of nudging and influencing behaviour in health care industry. We start with explaining the neoclassical discounted utility theory.

\section{Discounted Utility Theory}

The discounted utility model (DU model), as a framework, has been fundamental in the advancement of our knowledge concerning intertemporal choice. The model's central feature is that all future utilities discounted by the individual are consolidated into a single parameter. The DU model witnessed a rise in its popularity due to its simplicity, as well as the inclusion of the familiar compound interest formula. Samuelson (1937) begins constructing the DU model by highlighting three key assumptions:

The first assumption states that 'Utility is uniquely measurable as, in consequence, is marginal utility.' In other words, it assumes that the total value of several outcomes is equal to the discounted total of the utilities in each period. Equation (1) below displays a mathematical representation of this axiom where ' $U$ ' and ' $x$ ' depict marginal 
utility and money income per unit time, respectively.

$$
U=U(x)
$$

The next assumption describes how 'during any specified period of time, the individual behaves so as to maximise the sum of all future utilities, they being reduced to comparable magnitudes by suitable time discounting.' That is, a person assesses new choices by integrating them with their current plan. This is shown by maximising the integral given in equation (2) where ' $b$ ' represents the end of a finite time period.

$$
J=\int_{0}^{b} V(, t) d t
$$

Third, 'individuals discount future utilities in some simple regular fashion which is known to us.' Therefore, the instantaneous utility function is constant for all periods. Equation (3) illustrates this assumption, where the value of $\pi$, is represented in equation (4) as a function of a constant value of the discount rate $(P)$

$$
\begin{gathered}
V(x, t)=U(x) e^{-\pi t} \\
\pi=\log e(1+P)
\end{gathered}
$$

The final assumption allows us to re-write equation (2) so it resembles the following.

$$
J=\int_{0}^{b} U(x) e^{-\pi t} d t
$$

Subsequently, experimental conditions are defined. Initially, the individual is given an amount of money ' $S$ ', of which he can draw at will. Any money not drawn acquires a compounded interest at a given rate. Finally, the individual must allocate all his expenditure so that nothing is left at the end of the period. Mathematically, this is done by maximising equation (5), so it resembles equation (6), where ' $r$ ' represents to return on unused balance.

$$
S=\int_{0}^{b} x(t) e^{-r t} d t
$$

Maximising equation (3), with respect to conditions implied in equations (4) and (5), using a Lagrange multiplier ' $\lambda$ ' gives the following function.

$$
\int_{0}^{b} U(x) e^{-\pi t} d t-\lambda\left[\int_{0}^{b} x(t) e^{-r t} d t-S\right]
$$

To ensure this function is a maximum, the integral signs are ignored, and it is differentiated with respect to ' $x$ ', to give equation (8), where $U^{\prime}$ represents the marginal utility of income.

$$
U^{\prime}() e^{-\pi t}-\lambda e^{-r t}=0
$$

Finally, this can be rewritten as follows, where $\lambda$ is a constant depending on ' $S$ '.

$$
U^{\prime}(x)=\lambda e^{(\pi-r) t}
$$

Using equation (9), we can solve for $\mathrm{x}$, assuming we know the form of the utility function. However, if we know $\mathrm{x}$ as a function of $t$, the form of the utility function can be attained easily. 
The imposition of axioms that assume individuals always act rationally is impractical and thus, the DU model has received a great deal of criticism. Furthermore, Samuelson (1937) acknowledges the deficiencies of the model and hence, is cautious in its presentation. For example, he concludes his findings by quoting 'any connection between utility as discussed here and any welfare concept is disavowed.' As a result, empirical research on intertemporal choice has highlighted the model's shortcomings. The next section explains three of these anomalies, namely: magnitude effect, sign effect and the delay-speed up asymmetry.

The magnitude effect describes how large amounts of money suffer less proportional discounting than smaller amounts. The sign effect is derived from loss aversion, a concept introduced by Tversky and Kahneman (1979) which illustrates how people are risk averse for gains but risk seeking for losses. Therefore, gains are discounted at a higher rate than losses. Benzion et al. (1989) confirm this assertion, concluding that discount rates are indeed smaller for losses than gains, following their intertemporal choice experiment.

The delay-speed up asymmetry was initially demonstrated by Loewenstein (1988). It describes how discount rates can be influenced by whether the change in delivery time of an outcome is framed as an acceleration, or delay, from a specific reference point.

The DU model fails to describe actual intertemporal choice behaviour due to the aforementioned anomalies and underline the serious questions regarding an individual's rationality. This is because in many intertemporal situations, individuals mistrust their own ability to make rational and optimal decisions in the long term. It seems a constant discount rate is not associated with actual behaviour. However, empirical research has demonstrated that a hyperbolic model, instead of an exponential model, may explain intertemporal preferences more clearly. Nevertheless, Samuelson (1937) clearly makes the case that the DU model should only be used as a theoretical ideal upon which additional ideas can be built upon.

\section{Choice Architecture}

Choice architecture is a phenomenon that attempts to enhance suboptimal decision-making without restricting choices or economic incentives, primarily using nudges. It reflects the fact that there are many ways to present a choice to the decision-maker, and that what is chosen often depends upon how the choice is presented.

As a medium through which behaviour can be influenced, the policy tools of nudges and in extension, choice architecture, have demonstrated their effectiveness at impacting behaviour (Ly and Soman, 2013). Their efficiency as tools can be attributed to the ease of implementation in comparison with the alternatives of regulation and economic incentives. However, the usefulness of nudges heavily depends on the conditions. Therefore, it is critical to analyse past evidence of success or failure with regards to past nudging policies.

Implementation of choice architecture is more likely to be beneficial when the four following assumptions have been satisfied. Firstly, freedom of choice must be possible and thus, individual preferences may vary. Secondly, economic incentives or penalties are not appropriate. Thirdly, with regards to the situation encountered, behaviour is affected by cognitive influences. Lastly, there must be an increasing alignment with current regulations and incentives. On the other hand, strategies involving choice architecture should be avoided in two instances: If the intended outcome of the acting nudge goes against an individual's natural intentions and preferences and if current economic incentives need to be changed to enhance alignment with policy goals (Ly and Soman, 2013).

Existing literature identifies two approaches when using elements of choice architecture in the formation of adequate policies. The first approach seeks to remediate the choice biases or provide incentives for better choices. The second approach accepts that biased choices are normative and structures health messages, choices, interventions, and contexts in ways that use biases to promote good choices and outcomes (Loewenstein et al., 2007).

In intertemporal settings, the choice architect encounters three issues. First, individuals tend to heavily discount future outcomes due to being myopic and preferring to receive positive outcomes early (Loewenstein and Elster, 1992). Second, uncertainty about the future can cause individuals' preferences for future outcomes to be distorted, causing miscalculations in outcome probabilities. Third, individuals usually overestimate how much time and money they may have in the future due to being overly optimistic regarding forthcoming outcomes (Kahnemann and Lovallo, 1993).

Concepts emanating from choice architecture provide tools to tackle intertemporal issues. Weber et al. (2007) try to solve these issues by accentuating delayed options, through the alteration of decision environments, with the objective of promoting more patience for delayed rewards. This strategy portrays Bickel et al.'s aforementioned 
approach towards policy design using choice architecture. The methodology was supported by Loewenstein's (1988) inference that reference points are applicable to intertemporal choice, by suggesting that: instead of integrating delayed consumption with current consumption, it seems individuals usually perceive future consumption options as deviations from a reference point. Furthermore, Fischer et al. (1999) propose that differences in valuations shift the individual's preferences in a way that is consistent with changes in intentions and the endowment effect.

A lack of conceptual and definitional clarity, with respect to choice architecture, complicates the implementation process. Nevertheless, solving issues of clarity usually requires simple solutions, hinting that rapid progress operationally is not beyond our reach.

\section{Libertarian Paternalism}

The oxymoronic phrase 'Libertarian Paternalism' describes conflicting principles since libertarians believe in freedom of choice, whereas paternalists oppose freedom and thus, dispute libertarianism. King (2015) claims that the libertarian aspect of this concept explains that people should be able to exercise full freedom with respect to decisions. Hence, they should have the ability to opt out of arrangements if it is beneficial. The paternalistic facet expresses how a private or public institution seeks to positively influence people's choices when it does not believe their own choices are optimal with regards to welfare (Sunstein, 2017). Furthermore, libertarian paternalists accept that paternalism is inevitable in most cases, as choosing to do 'nothing' is seldom an option.

Jolls et al. (1997) underline that individuals are prone to decisions that negatively impact their own welfare, due to shortcomings informationally and cognitively, affecting intertemporal self-control. However, it has been discovered that people favour choices that allow for a higher number of alternatives. Bown et al. (2003) coined this phenomenon the 'Lure of choice.' This illustrates how "the probability that a given option will be chosen is increased if it is part of a larger choice set, between which a single choice is made, than if it is offered in isolation." The disagreement that emerges from conflict between whether coercing welfare-maximising choices or adopting a default rule that mirrors what most people actually want, is a considerable obstacle that choice architects confront. As follows, if deciding on a choice is itself an economic good, then coercing choices can be assumed to be feasible. However, when a decision becomes too technically sophisticated, people tend to avoid undertaking a decision, as many alternatives increase the cost in terms of effort exertion. Under these circumstances, default rules gain traction as a possible strategy.

Resistance from anti-paternalists produces a multitude of objections towards libertarian paternalism. The following section questions three objections, namely: slippery slopes; mistrust of planner and if libertarian paternalism is satisfactory in certain cases.

The first objection conveys the risk of overreaching paternalistically, after initial acceptance. Sceptics fear the subtle enactment of more invasive forms of paternalism. For example, to reduce cigarette consumption, some governments have moved on from warning labels to more oppressive approaches, such as smoking bans in public places. Hence, the question of where the line is, in terms of invasiveness, becomes crucial. Thus, to avoid sliding down this uncertain slope, maybe it is best to stay away from the slope altogether.

Libertarians respond to the slippery slope objection by highlighting how some form of paternalism is inevitable, because regulations and rules must be established to avoid discord. Additionally, the libertarian feature ensures as much freedom as possible is granted to the decision makers, implying that, metaphorically, the slope steepness is reduced. Furthermore, those who argue against libertarian paternalism point out that choice architects themselves are imperfect and can suffer from self-control problems. Although, it can be reasoned that in most cases the designated choice architect is likely to be aware with regards to his own self-control.

The second objection stems from a mistrust in the ability and intention of the choice architect. Notwithstanding, an individual will always have to make these choices and through additional research, designated architects will be more well-informed. Also, most institutions in the developed world can carry out checks and balances to protect against potentially disruptive arrangements.

Self-control problems give reason to paternalists asserting that in many cases, libertarian paternalism is too restrictive. Besides, in certain situations it is always advantageous to repress freedom of choice because people choices are suboptimal. Mistakes and incorrect decisions are sometimes essential in the long term because that is how humans learn and evolve. 


\section{Concluding Remarks}

Enhancing how we understand irrational decision making, along with how to influence behaviour through simple interventions is fundamental with regards to public policy. Consequently, governments are starting to realise the benefits that can be accrued through using behavioural economics, and more specifically nudges, to inform their decisions. For example, the introduction of the Behavioural Insights Team in the UK has led to the identification of multiple 'nudges' that would allow the government to save over $£ 300$ million. Additionally, the affordability of this approach is visible from the fact that the team has achieved a return of more than 10 times on their own costs (Ly and Soman, 2013). The noticeable achievements of the UK team have induced the creation of similar teams around the world. For instance, the USA has witnessed the establishment of the 'Cornell Center for Behavioural Economics in Child Nutrition Program', with the aim of promoting healthy eating in school. Furthermore, other countries have also recognised the advantages of 'nudging' policies with agencies in their infancies such as Denmark, Singapore and Canada. .

Although, the advent of Randomised Controlled Trials (RCTs) has been pivotal in terms of enriching the potential of behavioural economics. Other traditional disciplines have already witnessed the benefits that RCTs can bring. For example, before 2000 , approximately $19 \%$ of studies in non-health journals used impact evaluation; compared to $47 \%$ between 2005 and 2009. (Cameron et al., 2016). RCTs' capability is derived from its ability to yield internally valid estimates, and it achieves this by focusing on selection and omitted variable biases. Rubin (1978) defines that the potential outcome of an intervention, is the difference in averages observed between the treatment and control group; summed with the difference in selection bias and treatment effect. This is mathematically represented below in equation (25).

$$
D=E\left[Y_{i}^{c}-Y_{i}^{C} \mid T\right]+E\left[Y_{i}^{c} \mid T\right]-E\left[Y_{i}^{c} \mid C\right]
$$

The impact of interventions is estimated by observing the difference in results between the control and treatment group. This is feasibly accomplished because the element of randomisation allows for the omission of selection bias effects. Furthermore, the average treatment effect can be exclusively associated with the intervention, due to controlling for both observed and unobserved confounding factors. An added benefit of RCTs is that it includes only one assumption, which is the 'Stable Unit Treatment Value Assumption (SUTVA). The implication of SUTVA emphasises that there is no inference between units, as well as the absence of variation in treatment. (Schwartz et al., 2012).

Even though it has been described as the 'gold standard' of impact evaluation, RCTs still suffer several issues, such as external validity and ethical problems. External validity drawbacks originate from the exclusion of general equilibrium effects. Furthermore, failure to account for spill over effects causes estimates to be internally invalid. Additionally, the ethical concerns emerge since participants are relinquish sovereignty when part of a trial that is approved by a third party. In the end, the agreement is that there is a collective need to grow knowledge, as build-up of research will enhance the reliability of RCTs.

\section{References}

Benzion, U., Ammon, R., \& Yagil, J. (1989). Discount rates inferred from decisions: An experimental study. Management Science, 35(3), 260-265. https://doi.org/10.1287/mnsc.35.3.270

Brown, N. J., Read, D., \& Summers, B. (2003). The lure of choice. Journal of Behavioural Decision Making, 16(4), 297-308. https://doi.org/10.1002/bdm.447

Cameron, D. B., Mishra, A., \& Brown, A. N. (2016). The growth of impact evaluation for international development: how much have we learned? Journal of Development Effectiveness, 8(1), 1-21. https://doi.org/10.1080/19439342.2015.1034156

Jolls, C., Sunstien, C. R., \& Thaler, R. (1997). A behavioural approach to law and economics. Stan. L. Rev., 50, 1471. https://doi.org/10.2307/1229304

Kahnerman, D., \& Lovallo, D. (1993). Timid choices and bold forecasts: a cognitive perspective on risk taking. Management Science, 39(1), 17-31. https://doi.org/10.1287/mnsc.39.1.17

King, D. (2015). Applying behavioural insights to challenges in health policy. Retrieved from https://spiral.imperial.ac.uk/bitstream/10044/1/28411/king-D-2015-PhD-Thesis.pdf

Loewenstein, G. F. (1988). Frames of mind in intertemporal choice. Management Science, 34(2), 200-214. 
https://doi.org/10.1287/mnsc.34.2.200

Loewenstein, G. F., \& Jon, E. (Eds.). (1992). Choice over time. Russell Sage Foundation.

Loewenstein, G. F., Brennan, T., \& Volpp, K. (2007). Asymetric paternalism to improve health behaviours. Jama, 298(20), 2415-2417. https://doi.org/10.1001/jama.298.20.2415

Ly, K., \& Soman, D. (2013). Nudging around the world. Retrieved from http://cd-www.rotman.utoronto.ca/media/files/Programs-and-Areas/behavioral-economics/Nudging20

Rubin, D. B. (1978). Bayesian inference for causal effects: The role of randomization. The Annals of statistics, 6(1), 34-58. https://doi.org/10.1214/aos/1176344064

Samuelson, P. (1937). A note on measurement of utility. The review of economic studies, 4(2), 155-161. https://doi.org/10.2307/2967612

Schwartz, S., Gatto, N. M., \& Campbell, U. B. (2012). Extending the sufficient component cause model to describe the Stable Unit Treatment Value Assumption. Epidemiologic Perspectives \& Innovations, 9(1). https://doi.org/10.1186/1742-5573-9-3

Strotz, R. H. (1955). Myopia and inconsistency in dynamic utility maximisation. The Review of economic Studies, 23(3), 165-180. https://doi.org/10.2307/2295722

Sunstein, C. R. (2017). Human Agency and Behavioural Economics: Nudging Fast and Slow. Springer. https://doi.org/10.1007/978-3-319-55807-3

Tversky A., \& Kahneman, D. (1981). The framing of decisions and the psychology of choice. Science, 211(4481), 453-458. https://doi.org/10.1126/science.7455683

Weber, E. U., Johnson, E. J., Milch, K., Chang, H., Brodscholl, J., \& Goldstein, D. (2007). Asymmetric discounting in intertemporal choice: a query theory account. Psychological Science, 18, 516-523. https://doi.org/10.1111/j.1467-9280.2007.01932.x 